\title{
In situ X-ray powder diffraction studies of the synthesis of graphene oxide and formation of reduced graphene oxide
}

\author{
Storm, Mie Møller; Johnsen, Rune E.; Norby, Poul
}

Published in:

Journal of Solid State Chemistry

Link to article, DOI:

10.1016/j.jssc.2016.05.019

Publication date:

2016

Document Version

Peer reviewed version

Link back to DTU Orbit

Citation (APA):

Storm, M. M., Johnsen, R. E., \& Norby, P. (2016). In situ X-ray powder diffraction studies of the synthesis of graphene oxide and formation of reduced graphene oxide. Journal of Solid State Chemistry, 240, 49-54. https://doi.org/10.1016/j.jssc.2016.05.019

\section{General rights}

Copyright and moral rights for the publications made accessible in the public portal are retained by the authors and/or other copyright owners and it is a condition of accessing publications that users recognise and abide by the legal requirements associated with these rights.

- Users may download and print one copy of any publication from the public portal for the purpose of private study or research.

- You may not further distribute the material or use it for any profit-making activity or commercial gain

- You may freely distribute the URL identifying the publication in the public portal 


\section{Author's Accepted Manuscript}

In situ X-ray powder diffraction studies of the synthesis of graphene oxide and formation of reduced graphene oxide

Mie Møller Storm, Rune E. Johnsen, Poul Norby

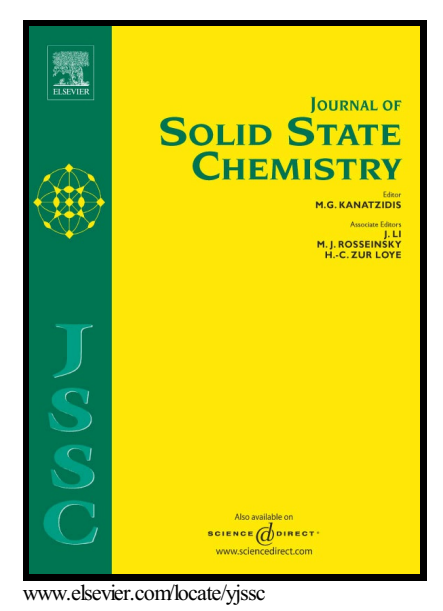

PII: $\quad$ S0022-4596(16)30199-2

DOI: $\quad$ http://dx.doi.org/10.1016/j.jssc.2016.05.019

Reference: $\quad$ YJSSC19404

To appear in: Journal of Solid State Chemistry

Received date: 10 March 2016

Revised date: 3 May 2016

Accepted date: 17 May 2016

Cite this article as: Mie Møller Storm, Rune E. Johnsen and Poul Norby, In sit X-ray powder diffraction studies of the synthesis of graphene oxide an formation of reduced graphene oxide, Journal of Solid State Chemistry http://dx.doi.org/10.1016/j.jssc.2016.05.019

This is a PDF file of an unedited manuscript that has been accepted fo publication. As a service to our customers we are providing this early version o the manuscript. The manuscript will undergo copyediting, typesetting, an review of the resulting galley proof before it is published in its final citable form Please note that during the production process errors may be discovered whic could affect the content, and all legal disclaimers that apply to the journal pertain 
In situ X-ray powder diffraction studies of the synthesis of graphene oxide and formation of reduced graphene oxide

Mie Møller Storm* ${ }^{1}$, Rune E. Johnsen and Poul Norby

Department of Energy Conversion and Storage, Technical University of Denmark, Frederiksborgvej 399, 4000 Roskilde, Denmark

\section{Abstract}

Graphene oxide $(\mathrm{GO})$ and reduced graphene oxide $(\mathrm{rGO})$ are important materials in a wide range of fields. The modified Hummers methods, for synthesizing GO, and subsequent thermal reduction to rGO, are often employed for production of rGO. However, the mechanism behinds these syntheses methods are still unclear. We present an in situ X-ray diffraction study of the synthesis of GO and thermal reduction of GO. The X-ray diffraction revealed that the Hummers method includes an intercalation state and finally formation of additional crystalline material. The formation of GO is observed during both the intercalation and the crystallization stage. During thermal reduction of GO three stages were observed: GO, a disordered stage, and the rGO stage. The appearance of these stages depends on the heating ramp. The aim of this study is to provide deeper insight into the chemical and physical processes during the syntheses.

\footnotetext{
${ }^{1}$ Corresponding author*: phone 004540848383 E-mail: mmst@dtu.dk
} 
Keywords: Hummers method, Thermal reduction, graphene oxide, reduced graphene oxide, X-ray diffraction, in situ

\section{Introduction}

Graphene oxide (GO) and reduced graphene oxide (rGO), synthesised from GO, has a future in fields ranging from electronics to energy technologies.[1] Small changes in the GO synthesis affects properties such as water intercalation[2] and surface functionalities[3], whereas changes in the thermal reduction routes causes changes in the deoxygenation process.[4] As small changes in the synthesis affects the product, thorough knowledge of the reactions could influence the choice in synthesis methods depending on the use of the material.

Graphene oxide was prepared as early as 1859 by Brodie.[5] Staudenmaier,1898[6] and Hummer and Offeman, 1958[7] proposed their own synthesis routes to GO formation, which improved the chemical safety and reduced the evolution of toxic gasses. Different modifications to the oxidation process have been made on the Hummers method[8-10], and these are now often known as modified Hummers methods. Even though several different variations of this process have been employed, the foundation of the methods remains the same: Concentrated sulfuric acid, permanganate and possibly nitrate form a highly oxidizing environment, which partially oxidize graphite into graphene layers with oxygen functionalities and defects.[11] The final step includes termination of the synthesis by $\mathrm{H}_{2} \mathrm{O}_{2}$ and water addition.

Even though the detailed structure of GO is still unknown, GO may be used in a range of applications $[12,13]$, as well as the more commonly known function as a possible precursor for large scale synthesis of the graphene-like material reduced graphene oxide (rGO).[1, 14-16] 
Dreyer et al[16] suggested that the active oxidizing species in the oxidation is dimanganese heptoxide, created by the reaction of permanganate and sulfuric acids.

$\mathrm{Mn}^{\mathrm{VII}} \mathrm{O}_{4}^{-}+3 \mathrm{H}_{2} \mathrm{SO}_{4} \rightarrow \mathrm{Mn}^{\mathrm{VII}} \mathrm{O}_{3}^{+}+\mathrm{H}_{3} \mathrm{O}^{+}+3 \mathrm{HSO}_{4}{ }^{-}$

$\mathrm{MnO}_{3}{ }^{+}+\mathrm{MnO}_{4}{ }^{-} \rightarrow \mathrm{Mn}_{2} \mathrm{O}_{7}$

This suggestion was based on $\mathrm{Mn}_{2} \mathrm{O}_{7}$ ability to selectively oxidize unsaturated aliphatic double bonds, compared to aromatic bonds. The presence of this species has yet to be confirmed.

Dimiev and Tour[17] investigated samples obtained during the modified Hummers method by optical microscopy and Raman spectroscopy. Centrifuged and separated samples were analysed by X-ray diffraction (XRD). They observed three stages during the modified Hummers method; graphite intercalation, graphite oxidation and formation of GO. Kang et al[18] observed that the addition of water during the termination of the synthesis also affect the GO product, showing how small changes in the synthesis affects the results.

Different reduction methods have been used for the transformation of GO to rGO. Chemical or thermal reduction are common synthesis routes towards rGO. Chemical reduction by hydrazine removes many of the oxygen functionalities and introduces nitrogen atoms to the rGO.[19] Other variation in the methods and other chemical reduction methods have been successfully employed as well and has been described in various reviews along with the many properties of rGO.[11, 16, 20] That GO undergoes changes upon heating was observed by Brodie, as a reduction of the oxygen and hydrogen components compared to carbon was observed when GO was heated.[5] Thermal reduction leads to decomposition and expansion of the $\mathrm{GO}$ layers by formation of $\mathrm{CO}_{2}, \mathrm{CO}$ and $\mathrm{H}_{2} \mathrm{O}$ gas, [21] which causes pressure between the carbon planes in GO. The change from ordered GO to a more amorphous rGO upon shock heating is clearly observed by XRD studies where GO, with a sharp 001 diffraction peak, is changed to disordered $\mathrm{rGO}[22]$. Thermal reduction of GO causes structual damage and lattice defects to the 
graphene plane as carbon atoms are removed.[11] Thermal reduction can be performed in various atmospheres and at different heating rates.[20] Few in situ studies of the formation of rGO have been made. Osswald et al. [23] studied the reduction expansion synthesis of GO with urea to graphene with in situ Raman spectroscopy from $25-800{ }^{\circ} \mathrm{C}$. The $\mathrm{G}$ band and the corresponding full width at half maximum changed upon reaction indicating clear irreversible changes. The $\mathrm{G}$ band was downshifted which can be caused by changes in the oxygen surface groups, healing of structural defects or changes in interlayer spacing. A minimum temperature of $800{ }^{\circ} \mathrm{C}$ was needed during the synthesis with urea as expansion agent.

In this paper we present results from in situ XRD experiments during GO synthesis by the modified Hummers method and the thermal reduction of GO with both reactions being performed in capillary cells. The modified Hummers methods shows new diffraction peaks for GO and the thermal reduction reveals a new stage in the transition between GO and rGO.

\section{Experimental}

\subsection{Ex situ synthesis of GO:}

$3 \mathrm{~g}$ natural flake graphite (325 mesh, $98 \%$ metal basis supplied by Alfa Aesar) was mixed with $1.5 \mathrm{~g}$ $\mathrm{NaNO}_{3}$ (Alfa Aesar) in an Erlenmeyer flask on ice. $100 \mathrm{ml}$ conc. $\mathrm{H}_{2} \mathrm{SO}_{4}$ (Sigma Aldrich) was added under stirring, and after $10 \mathrm{~min}$ with ice bath cooling $12.0 \mathrm{~g} \mathrm{KMnO}_{4}$ (Alfa Aesar) was added over a time period of $40 \mathrm{~min}$. The dispersion was cooled on ice for $2 \mathrm{hrs,} \mathrm{before} \mathrm{the} \mathrm{reaction} \mathrm{mixture} \mathrm{was}$ heated in a water bath to $35^{\circ} \mathrm{C}$. Samples with reaction times of $3 \mathrm{hrs}$ and 3 days were prepared. A sample of the mixture was extracted for ex situ XRD after heating for $3 \mathrm{hrs}$ and stored in a small Duran laboratory bottle (named the GO-intermediate). The Erlenmeyer flask and the small extracted sample were placed on ice to terminate the reaction. The reaction mixture was diluted with $100 \mathrm{ml}$ ice cooled 
water which was followed by slow addition of $25 \mathrm{ml} \mathrm{H}_{2} \mathrm{O}_{2}(30 \% \mathrm{w} / \mathrm{w}$ in water, from Sigma Aldrich). The GO powder was isolated by centrifugation at $4500 \mathrm{rpm}$ for $10 \mathrm{~min}$, and the precipitate was washed with $0.1 \mathrm{M} \mathrm{H}_{2} \mathrm{SO}_{4}$ and dried in a vacuum oven at $25{ }^{\circ} \mathrm{C}$ overnight. The $\mathrm{GO}$ was washed four times with $1 \mathrm{M} \mathrm{HCl}$ with centrifugation and dried in a vacuum oven overnight at $25^{\circ} \mathrm{C}$. This washing process was repeated with acetone.

2.2 The in situ modified Hummers method:

$0.15 \mathrm{~g}$ Alfa Aesar graphite was mixed with $5 \mathrm{ml}$ conc. $\mathrm{H}_{2} \mathrm{SO}_{4}$ by magnetic stirring for approx. 1 day, forming a graphite suspension. A quartz glass capillary cell ( $0.7 \mathrm{~mm}$ outer diameter) was prepared having quartz wool in one end, followed by $5 \mathrm{~mm}$ of loosely packed carefully grinded $\mathrm{KMnO}_{4}$ powder. Approximately $7 \mathrm{~mm}$ graphite suspension was placed with a $5 \mathrm{~mm}$ gap to the $\mathrm{KMnO}_{4}$ powder in the capillary (Figure 1). FigureS1, in supplementary material, shows a picture of the actual set-up. The capillary was mounted in a Swagelok based gas inlet system with a Vespel/graphite ferrule; a rather similar setup was described in [24]. The capillary was aligned in the $\mathrm{X}$-ray beam on the $\mathrm{KMnO}_{4}$ powder for X-ray measurements with an exposure time of $30 \mathrm{~s}$. The XRD data collection was initiated and the graphite suspension was mixed with the $\mathrm{KMnO}_{4}$ powder by gently adding a pressure of $\mathrm{N}_{2}$ gas inside the capillary. Similar in situ experiments were performed three times at the synchrotron, with similar results.

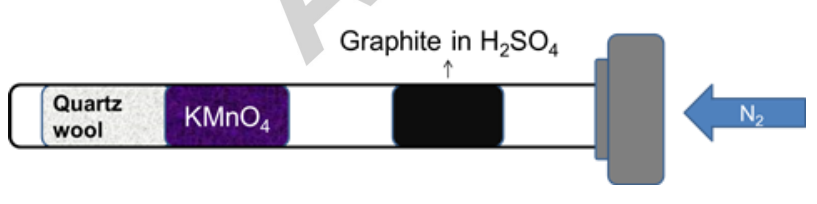

Figure 1: The capillary set-up for the graphite oxidation.

2.3 In situ GO thermal reduction: 
A quartz glass capillary ( $0.7 \mathrm{~mm}$ outer diameter) was filled with quartz wool plugs in both ends with GO between the wool plugs. The GO was synthesized by the modified Hummers method with oxidation time of $3 \mathrm{hrs}$ or 3 days, as described above.[3] Unless otherwise stated the GO powder used during the synthesis was the GO powder with an oxidation time of $3 \mathrm{hrs}$. The capillary was open in both ends and mounted in a modified Swagelok cell using a Vespel/graphite ferrule and connected to a gas delivery system. A small constant flow of $\mathrm{N}_{2}$ gas was applied before the heating was initiated. The samples were measured with an exposure time of $30 \mathrm{~s}$ and different temperature ramps of 1, 5, 10, 20 and $50^{\circ} \mathrm{C} / \mathrm{min}$. A hot-air blower was used to increase the temperature from 25 to $690^{\circ} \mathrm{C}$, and data were also collected during cooling. The temperature of the hot-air blower was calibrated using the known thermal expansion of metallic silver. A powdered silver sample in a capillary was heated and the unit-cell parameters were determined using Rietveld refinement as a function of the settemperature. From these data a calibration curve was determined, allowing the actual sample temperature to be estimated. In order to decrease the effect of thermal gradients in the capillary, the Xray beam is kept much smaller than the width of the hot-air blower. We believe that the calibrated temperatures are correct to within a few degrees. The hot-air blower had a non-linear increase in temperature despite its settings, as seen from the y-axis in Figure 5.

\subsection{X-ray diffraction:}

2.4.1 Synchrotron XRD: X-ray powder diffraction data were collected at the Swiss Norwegian beamline (SNBL), ESRF, with a wavelength of $0.7020 \AA$, a beam size of $0.5 \times 0.5 \mathrm{~mm}$ and a sample-todetector distance of $199.72 \mathrm{~mm}$. The data were integrated by Fit2D[25] and normalized to a small $2 \theta$ range of the background (3.4-3.8 in $2 \theta$ for the GO synthesis and $24-27^{\circ}$ in $2 \theta$ for the thermal 
reductions) in Powder3D[26]. Peak positions, intensity and d-values of in situ data was analyzed by Diffract suite EVA (Bruker software).

The graphite diffraction peaks described in the result and discussion section are dominantly from graphite $2 \mathrm{H}$.

2.4.2 Ex situ XRD: X-ray powder diffraction patterns of the GO-intermediate sample were collected on a Rigaku Smartlab X-ray diffractometer (Cu-Ka radiation) with a step size of 0.02 in $2 \theta$ at $1 \% \mathrm{~min}$ from 5-120 in $2 \theta$ using Bragg-Brentano geometry and in transmission geometry (focusing optics) for the capillary data $\left(5-90^{\circ}\right.$ in $2 \theta$, step size $0.02^{\circ}$ in $\left.2 \theta\right)$. XRD measurements of the GO-intermediate sample were performed after synthesis on an open sample holder. After 10 days confinement of the sample in a Duran laboratory glass bottle, the sample was measured in a capillary. The XRD diffraction patterns were similar.

3. Results and discussion

3.1 The modified Hummers method

Figure 2 shows the XRD patterns of the modified Hummers method of GO synthesis in the capillary setup, which displayed three different stages. The intensity of the measured diffraction patterns decreased strongly during stage II, as seen in Figure 2. This might be related to movements of the solvent in the capillary.

The first changed observed in the XRD patterns (termed stage I) consisted of the dissolution of $\mathrm{KMnO}_{4}$. This was succeeded by an intercalation of the synthesis mixture into graphite (stage II) which was followed by formation of additional crystalline material (stage III). The GO was observed early 
during the synthesis by the 001 diffraction peak $^{2}$ at $5.01^{\circ}$ in $2 \theta, d=7.97 \AA$, which increased in intensity through both stage II and III. This shows how GO is formed from the very beginning of the synthesis and continues to be formed during the synthesis. The last stage III of the modified Hummers methods show GO formation along with new previously unobserved diffraction peaks assigned to GO. The additional diffraction peaks of GO are not observed in the final product and reveals an ordered structure for GO during synthesis. Furthermore an additional unidentified new crystalline compound is observed in stage III as well.

\footnotetext{
${ }^{2}$ For GO the patterns are indexed based on a hexagonal unit cell, where the length of the $c$-axis is equal to the interlayer distance.
} 


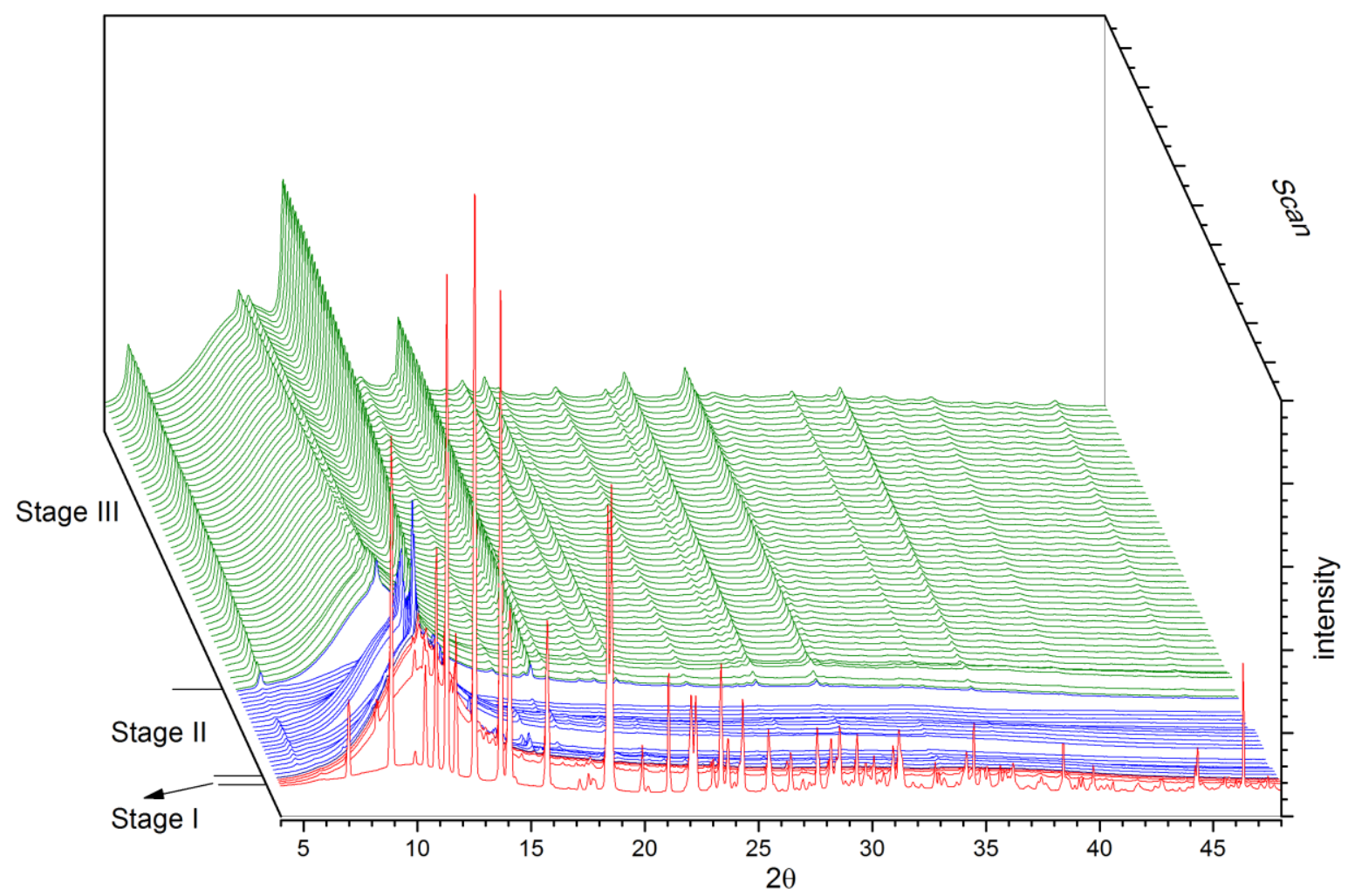

Figure 2: The in situ XRD patterns for the GO capillary synthesis. Stage I displays every scan, stage II every $2^{\text {nd }}$ and stage III every $3^{\text {rd }}$.

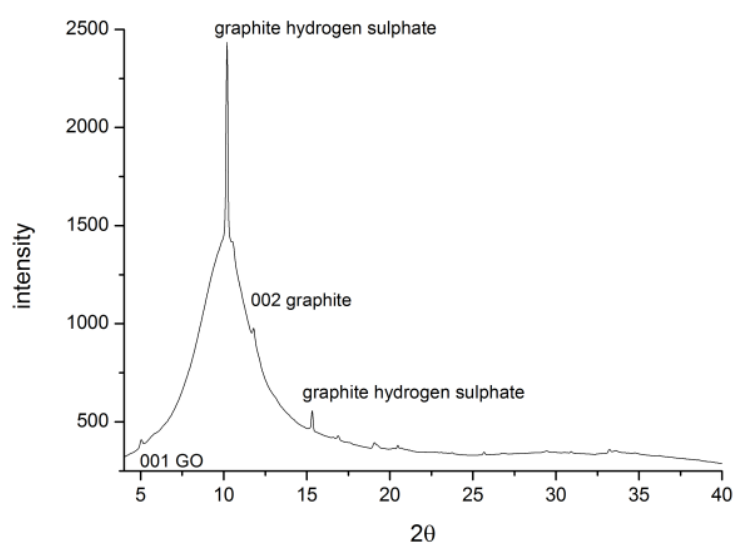

Figure 3: Stage II of the GO synthesis. 
Stage II displayed few diffraction peaks as seen in Figure 3 (assignment in Table S1). During stage II peaks which may be assigned to graphite-hydrogensulphate appeared.[27] Graphite-hydrogensulphate is further discussed in SI. These diffraction peaks could indicate that the modified Hummers method have an intercalation of sulfuric acid or hydrogensulphate into the graphite layers, followed by further oxidation and water intercalation upon the GO formation. The sharp diffraction peak at $10.19^{\circ}$ in $2 \theta$ during stage II has a small high-angle shoulder at $10.49^{\circ}$ in $2 \theta$ which may be indexed as the 002 diffraction peak of GO.

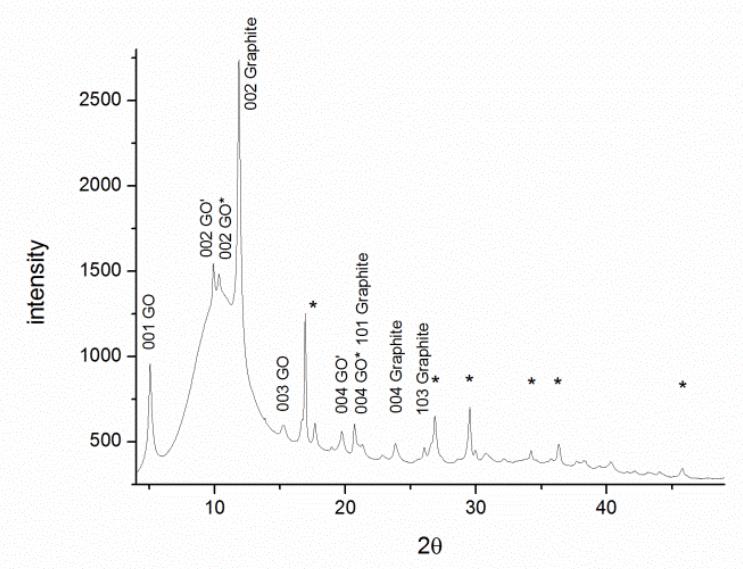

Figure 4: Stage III of the GO synthesis, showing a split of the GO diffraction peaks, here termed GO* and GO', the calculated GO diffraction peak is placed between the double peaks. *mark the cubic reflections

The diffraction peaks and the assignment of diffraction peaks of stage III for the in situ data can be seen in Figure 4 and Table S2. Most peaks were identified but a complete assignment of the diffraction peaks for stage III was not possible. The diffraction peaks assigned to graphite in Figure 4 are discussed in Supplementary material (SI). 
For stage III several diffraction peaks were observed which can be ascribed to GO. A splitting of the GO diffraction peak was observed for the in situ diffraction patterns (Figure 4 shows some of the diffraction peaks assigned as GO' or GO*). The split GO diffraction peaks have not been reported for ex situ XRD patterns of synthesized and washed GO. The expected and calculated d-value of GO(001) lies between the observed GO' and GO* d-values. The splitting of these diffraction peaks could be caused by different solvent intercalation. The splitting of the GO peaks during synthesis are not observed in the solid samples (see Figure 6A for a GO diffractogram). The reduced number of diffraction peaks observed in the solid GO could be explained from elimination of solvent interaction by washing.

It was not possible to identify any compounds related to the other diffraction peaks at stage III, however a number of diffraction peaks (see Figure 4) can be indexed based on a cubic unit cell with $\mathrm{a}=3.37 \AA$. It has been suggested that $\mathrm{Mn}_{2} \mathrm{O}_{7}$ plays a major role in the Hummers synthesis[17], but as $\mathrm{Mn}_{2} \mathrm{O}_{7}$ is liquid at room temperature it cannot be observed by diffraction. It is worth noting that $\mathrm{Mn}_{2} \mathrm{O}_{7}$ is often formed as a highly explosive dark-green oil.

The XRD pattern of the laboratory GO-intermediate sample (which was extracted after the oxidation of graphite before termination of the experiment and measured "as is") resembled the XRD pattern obtained in situ at stage III in a capillary, with more well defined peaks, indicating that no significant changes were caused by the confined space of the capillary sample or the addition of $\mathrm{NaNO}_{3}$ in the ex situ synthesis. The diffraction pattern of stage III, measured ex situ, can be indexed based on a triclinic unit cell. The unit cell was found by indexing and profile refinement to have the following parameters: $a=4.763 \AA, b=8.268 \AA, c=4.711 \AA, \alpha=105.5^{\circ}, \beta=119.4^{\circ}$ and $\gamma=74.6^{\circ}$, unit cell volume $154.65 \AA^{3}$. The profile fit can be seen in supplementary material Figure S2. Care must be taken to validate the suggested unit cell when a structure is indexed as triclinic, but all peaks were indexed and only some 
calculated peaks were not observed. The triclinic unit cell has some similarities to the hexagonal unit cell of GO with a $\beta$-angle close to $120^{\circ}$ and a close to $\mathrm{c}$. It was not possible to find a known crystal structure with similar triclinic unit cell parameters. Further investigations are needed to elucidate the structure and composition of this phase in the modified Hummers synthesis.

\subsection{Thermal reduction of graphene oxide}

The GO thermal reduction goes through three stages, clearly observed from the changes in the initial 001 GO diffraction peak ( $4.9^{\circ}$ in $2 \theta, d=8.20 \AA$ ). See Figure 5 where the evolution in the diffraction pattern during heating at $5{ }^{\circ} \mathrm{C} / \mathrm{min}$ is shown. The trend is similar for the heating ramps of 1,10 and 20 ${ }^{\circ} \mathrm{C} / \mathrm{min}$. For a 3D plot of the GO reduction at $5{ }^{\circ} \mathrm{C} / \mathrm{min}$ see Figure S3. The in situ XRD shows three stages: a GO stage, a disordered stage and an ordered rGO stage. The disordered stage is observed for both high and low temperature ramps for heating of GO and has, to our knowledge, not been identified before. The low temperature disordered stage appear for all temperature ramps indicating that even at slow heating a disordered transition stage is involved. Reordering of rGO may take place during the heating depending on the ramp rate. The disordered stage has a resemblance to rGO formed by shock heating, while it has previously been assumed that the graphene layers of GO were ripped apart only upon fast heating. 


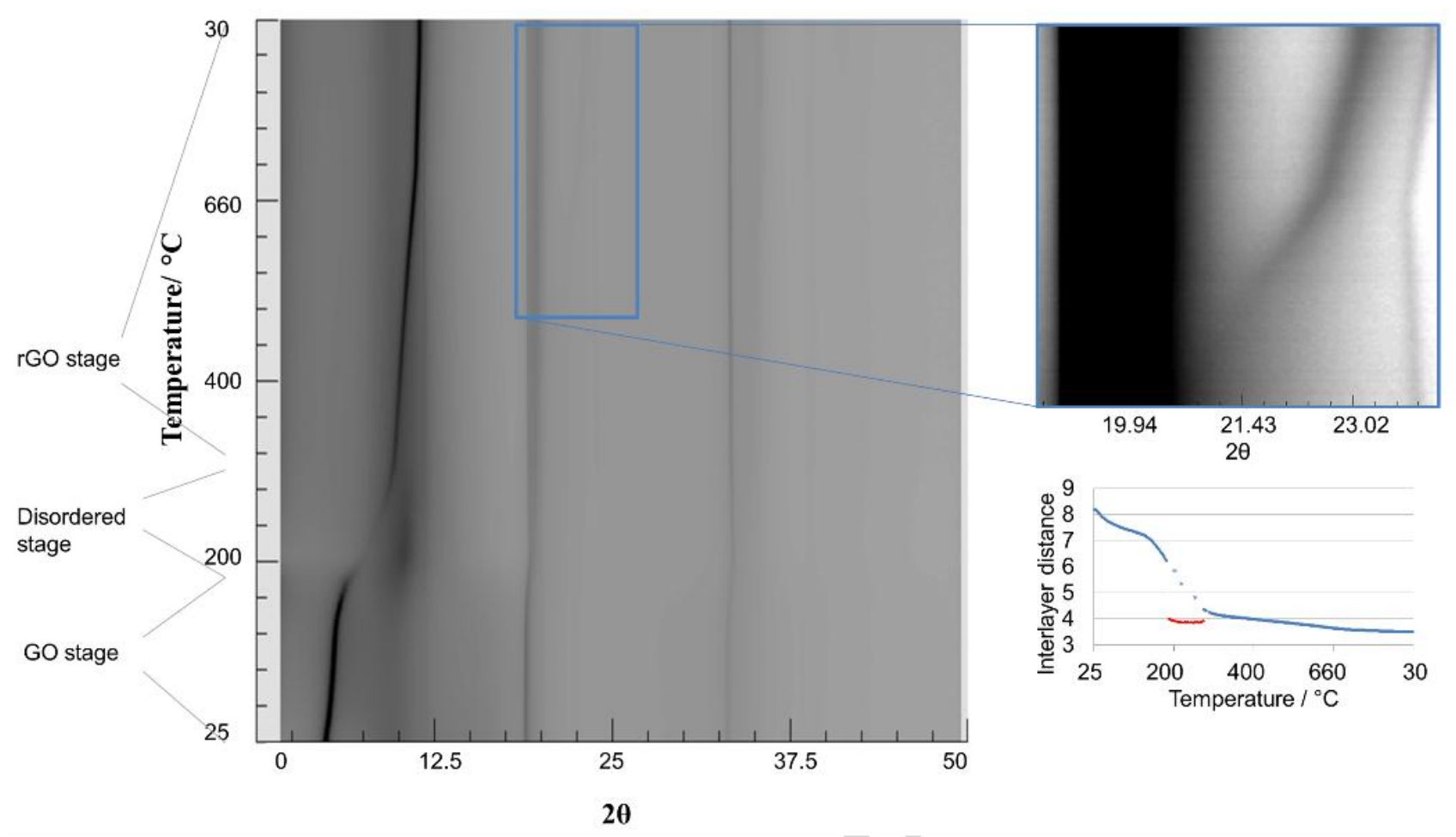

Figure 5: A 2D plot of diffraction patterns during transformation of GO to $\mathrm{GGO}$ measured from $10-50^{\circ}$ in $2 \theta$, for the $5^{\circ} \mathrm{C} / \mathrm{min}$ heating rate, the approximate duration of the $\mathrm{GO}$, disordered and rGO stage have been indicated. Inset: The appearance of a small peak at $22.5^{\circ}$ in $2 \theta$. Right, below: Plot of the interlayer distance vs. temperature, the dots connecting the two solid blue lines indicate the estimated d-spacing of the weak diffraction peak connecting the GO and rGO stage.

Figure 5 shows that the interlayer distances in GO decreased as the temperature increased, probably due to evaporation of intercalated water and partial reduction. Just below $200{ }^{\circ} \mathrm{C}$ the intensity of the 001 reflection decreased sharply and a broad peak appeared (at a d-value of approx. $4 \AA$, marked with red in the plot of temperature vs interlayer distance in Figure 5) indicating formation of a disordered phase (here named the disordered stage). However, the 001 reflection did not disappear completely; a low intensity contribution from the 001 reflection continued to move toward lower d-values as seen in 
Figure 5 and eventually reached the rGO stage. The 100 and 110 reflections of GO were observed during the disordered stage, see Figure 5 and Figure 6A. The rGO phase was formed upon further heating, as the broad peak of the disordered stage changed into a more well-defined peak with d-values similar to the 002 diffraction peak observed for rGO.[28] The 002 reflection from graphite was present during the entire experiment as an impurity in the GO material. The position of the $002 \mathrm{rGO}$ diffraction peak ( $\mathrm{d}=3.50 \AA$, after cooling) during growth did not reach the d-value of the graphite reflection $\left(\mathrm{d}=3.37 \AA\right.$ ) for the 5 and $20^{\circ} \mathrm{C} / \mathrm{min}$ experiments, indicating an incomplete reduction. For the controlled temperature ramp of 1 and $10^{\circ} \mathrm{C} / \mathrm{min}$ the interlayer distance of the rGO phase ended up closer to the 002 graphite diffraction peak after cooling ( $(\mathrm{d}=3.43 \AA$ ) , indicate more complete conversion to graphite. The stages of the thermal reduction can be separated by grouping of the interlayer distance, as seen in Figure 5. The GO interlayer distance decreased from 8.2 to $6.4 \AA$, the disordered stage is below $4 \AA$ and the rGO stage decreased from 4.5 to $3.5 \AA$.

The three stages, the GO stage, the disordered stage and the rGO stage, were observed during heating of GO with temperature ramps of $1,5,10$ and $20^{\circ} \mathrm{C} / \mathrm{min}$. The diffraction peaks of the GO and the rGO stages were assigned as seen in Figure 6 (The diffraction peaks of the rGO stage are given in Table S3 and the GO diffraction peaks are described in SI). 

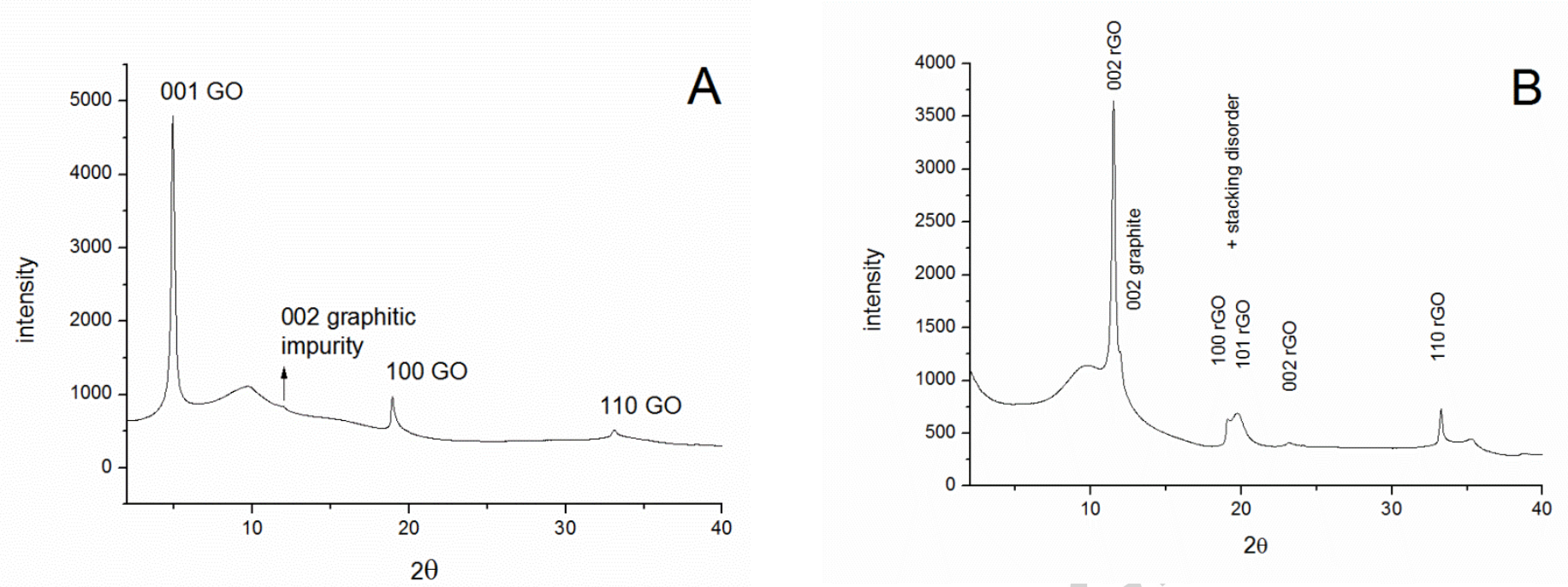

Figure 6: A: A diffraction pattern of the initial GO stage of the thermal reduction of GO, B: A diffraction pattern of the rGO stage of the thermal reduction of GO.

The on-set temperature of the disordered stage varied with temperature ramp rate. The disordered stage was observed in the same temperature range for the 5,10 and $20^{\circ} \mathrm{C} / \mathrm{min}$ temperature ramps, from approximately $210^{\circ} \mathrm{C}$ to $285^{\circ} \mathrm{C}$. The $1{ }^{\circ} \mathrm{C} / \mathrm{min}$ temperature ramp displayed the disordered stage from approximately $190^{\circ}$ to $230^{\circ} \mathrm{C}$. Decreasing the temperature ramping rate lowers the transition temperature due to thermally activated processes.

At increased temperature ramping rate of $50{ }^{\circ} \mathrm{C} / \mathrm{min}$ a decrease of the diffraction intensity of the 002 rGO reflection was observed, see Figure 7. Visual observation showed that some of the GO material had moved in the capillary, most likely due to the rapid expansion during thermal reduction. However, as the 002 graphite reflection was observed with a rather constant intensity throughout the entire scan series we assume that the movement did not affect the amount of material in the X-ray beam. The low 
intensity $002 \mathrm{rGO}$ peak (at $11.5^{\circ}$ in $2 \theta$, see Figure 7) indicates that almost all of the synthesized rGO was disordered at this high temperature ramp.

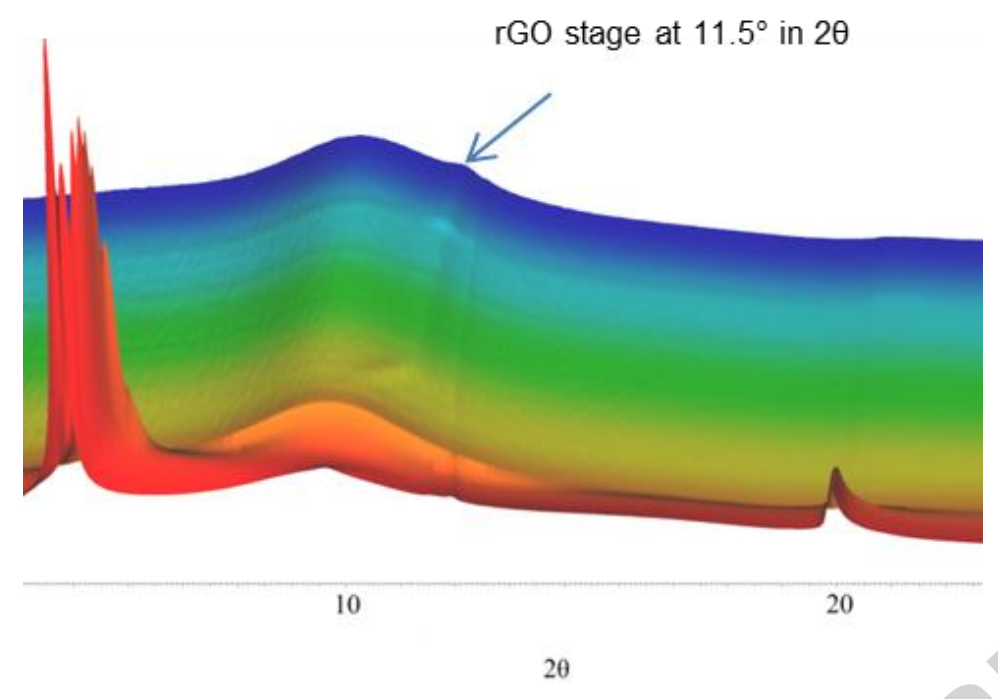

Figure 7: XRD 3D surface plot of the thermal reduction of $\mathrm{GO}$ at $50{ }^{\circ} \mathrm{C} / \mathrm{min}$.

Thermal reduction of GO, with the GO inserted directly into a furnace at around $1000{ }^{\circ} \mathrm{C}$, resulted in a similar very broad or absent $002 \mathrm{rGO}$ peak. It is plausible that a high heating rate and the rapid decomposition inhibit the formation of a more ordered material. The formation of an amorphous product upon shock heating of GO was also observed by McAllister et al[22], whereas they also observed a sharp diffraction peak and a high order $\mathrm{rGO}$ when the $\mathrm{GO}$ was heated at $1{ }^{\circ} \mathrm{C} / \mathrm{min}$. They suggested that the diffusion of the evolved gases during the synthesis was sufficient slow, for slower heated samples, to avoid exfoliation of the graphene oxide thus forming the ordered rGO.

Our rGO results show similar XRD pattern as those which were made in regular furnace. However as we perform in situ XRD we observe the formation of a disordered phase at an earlier stage in the reduction. This could suggest that the smaller pressure in the slowly heated rGO is not the full 
explanation for the formation of the ordered rGO. The lower pressure may enable rGO to form a relative ordered layered structure, revealing in the observed sharp 002 rGO diffraction peak, but as some order is re-gained also for the fast heated samples, this indicate that even samples which have been ripped apart may re-order to some degree. However, as our results are obtained by reaction in a capillary they could differ from what would be obtained in a furnace.

The three different stages for the thermal reduction of GO was also observed for a more oxidized sample GO sample (3 days oxidation time), details in SI. The observation of the same stages indicates that formation of a disordered stage is a common occurrence for all GO samples.

The disordered stage was observed for all the different reductions of GO which opens up for considerations of possible isolation of the disordered stage as a separate compound from low temperature treatment.

\section{Conclusions}

In situ XRD diffraction data for the modified Hummers method and thermal reduction of GO performed in a capillary were obtained. The oxidation by the modified Hummers method started with intercalation where sulfuric acid and $\mathrm{HSO}_{4}{ }^{+}$was intercalated into the graphite. Formation of GO was observed concomitant with the intercalation and GO formation was continued into the last stage. In the last stage new diffraction peaks from GO during synthesis appeared, which were not observed in dried and cleaned GO. Stage III furthermore showed the development of a possible GO related crystalline material. Thermal reduction of GO to rGO occurs over three different stages. Small changes in the interlayer distance of GO were observed during the first stage with the d-value decreasing upon heating. The second disordered stage was observed at all heating rates. The temperature rate depended 
on the heating rate. following the disordered stage formation of ordered rGO was observed by XRD.

However, at high heating rates the final rGO stage is more disordered than at lower heating rates. The observation of a disordered stage for all the investigated thermal reductions of GO indicate that the final rGO product results from reordering of the graphene layers.

Acknowledgement

The authors acknowledge and thanks for the support given by the staff at the Swiss-Norwegian beamline, ESRF, the ReLiable project funded by the Danish Council for Strategic Research-Programme Commission on Sustainable Energy and Environment (project no. 11-116792), and The Danish Research Council for the financial support of synchrotron measurements (DANSCAT).

Supplementary Material Available

Supplementary material contains pictures of experimental set-up, additional 2D XRD and peak assignments. This material is available free of charge via the Internet

\section{References}

[1] M. Segal, Selling graphene by the ton, Nat Nano, 4 (2009) 612-614.

[2] T.N. Blanton, D. Majumdar, X-ray Diffraction Characterization of Polymer Intercalated Graphite Oxide, Powder Diff., 27 (2012) 104-107.

[3] M.M. Storm, M. Overgaard, R. Younesi, N.E.A. Reeler, T. Vosch, U.G. Nielsen, K. Edström, P. Norby, Reduced graphene oxide for $\mathrm{Li}$-air batteries: The effect of oxidation time and reduction conditions for graphene oxide, Carbon, 85 (2015) 233-244.

[4] A. Ganguly, S. Sharma, P. Papakonstantinou, J. Hamilton, Probing the Thermal Deoxygenation of Graphene Oxide Using High-Resolution In Situ X-ray-Based Spectroscopies, J. Phys. Chem. C, 115 (2011) 17009-17019.

[5] B.C. Brodie, On the Atomic Weight of Graphite, Philosophical Transactions of the Royal Society of London, 149 (1859) 249-259.

[6] L. Staudenmaier, Verfahren zur Darstellung der Graphitsäure, Berichte der deutschen chemischen Gesellschaft, 31 (1898) 1481-1487. 
[7] W.S. Hummers, R.E. Offeman, Preparation of Graphitic Oxide, J. Am. Chem. Soc., 80 (1958) 1339-1339. [8] D.C. Marcano, D.V. Kosynkin, J.M. Berlin, A. Sinitskii, Z. Sun, A. Slesarev, L.B. Alemany, W. Lu, J.M. Tour, Improved Synthesis of Graphene Oxide, ACS Nano, 4 (2010) 4806-4814.

[9] N.I. Kovtyukhova, P.J. Ollivier, B.R. Martin, T.E. Mallouk, S.A. Chizhik, E.V. Buzaneva, A.D. Gorchinskiy, Layerby-Layer Assembly of Ultrathin Composite Films from Micron-Sized Graphite Oxide Sheets and Polycations, Chem. Mater., 11 (1999) 771-778.

[10] M. Hirata, T. Gotou, S. Horiuchi, M. Fujiwara, M. Ohba, Thin-film particles of graphite oxide 1: High-yield synthesis and flexibility of the particles, Carbon, 42 (2004) 2929-2937.

[11] S. Pei, H.-M. Cheng, The Reduction of Graphene Oxide, Carbon, 50 (2012) 3210-3228.

[12] K.P. Loh, Q. Bao, G. Eda, M. Chhowalla, Graphene oxide as a chemically tunable platform for optical applications, Nat Chem, 2 (2010) 1015-1024.

[13] D. Chen, H. Feng, J. Li, Graphene Oxide: Preparation, Functionalization, and Electrochemical Applications, Chem. Rev., 112 (2012) 6027-6053.

[14] L. Zhang, J.J. Liang, Y. Huang, Y.F. Ma, Y. Wang, Y.S. Chen, Size-controlled synthesis of graphene oxide sheets on a large scale using chemical exfoliation, Carbon, 47 (2009) 3365-3368.

[15] Q.Q. Zhuo, J. Gao, M.F. Peng, L.L. Bai, J.J. Deng, Y.J. Xia, Y.Y. Ma, J. Zhong, X.H. Sun, Large-Scale Synthesis of Graphene by the Reduction of Graphene Oxide at Room Temperature Using Metal Nanoparticles as Catalyst, Carbon, 52 (2013) 559-564.

[16] D.R. Dreyer, S. Park, C.W. Bielawski, R.S. Ruoff, The Chemistry of Graphene Oxide, Chem. Soc. Rev., 39 (2010) 228-240.

[17] A.M. Dimiev, J.M. Tour, Mechanism of Graphene Oxide Formation, ACS Nano, 8 (2014) 3060-3068. [18] J.H. Kang, T. Kim, J. Choi, J. Park, Y.S. Kim, M.S. Chang, H. Jung, K.T. Park, S.J. Yang, C.R. Park, Hidden Second Oxidation Step of Hummers Method, Chemistry of Materials, 28 (2016) 756-764.

[19] S. Stankovich, D.A. Dikin, R.D. Piner, K.A. Kohlhaas, A. Kleinhammes, Y. Jia, Y. Wu, S.T. Nguyen, R.S. Ruoff, Synthesis of Graphene-Based Nanosheets via Chemical Reduction of Exfoliated Graphite Oxide, Carbon, 45 (2007) 1558-1565.

[20] S. Mao, H. Pu, J. Chen, Graphene Oxide and Its Reduction: Modeling and Experimental Progress, RSC Advances, 2 (2012) 2643-2662.

[21] H.C. Schniepp, J.-L. Li, M.J. McAllister, H. Sai, M. Herrera-Alonso, D.H. Adamson, R.K. Prud'homme, R. Car, D.A. Saville, I.A. Aksay, Functionalized Single Graphene Sheets Derived from Splitting Graphite Oxide, J. Phys. Chem. B, 110 (2006) 8535-8539.

[22] M.J. McAllister, J.-L. Li, D.H. Adamson, H.C. Schniepp, A.A. Abdala, J. Liu, M. Herrera-Alonso, D.L. Milius, R. Car, R.K. Prud'homme, I.A. Aksay, Single Sheet Functionalized Graphene by Oxidation and Thermal Expansion of Graphite, Chem. Mater. 19 (2007) 4396-4404.

[23] M. Mowry, D. Palaniuk, C.C. Luhrs, S. Osswald, In situ Raman spectroscopy and thermal analysis of the formation of nitrogen-doped graphene from urea and graphite oxide, RSC Advances, 3 (2013) 21763-21775. [24] R.E. Johnsen, F. Krumeich, P. Norby, Structural and Microstructural Changes During Anion Exchange of CoAl Layered Double Hydroxides: An In Situ X-ray Powder Diffraction Study, J. Appl. Crystallogr., 43 (2010) 434447.

[25] A.P. Hammersley, FIT2D: An Introduction and Overview, ESRF Internal Report ESRF97HA02T (1997).

[26] B. Hinrichsen, R.E. Dinnebier, M. Jansen, Powder3D 1.2, DOI (2004) Max Planck Institute for Solid State Research.

[27] L. Monyakina, V. Avdeev, I. Nikol'skaya, A. Litvinenko, N. Fadeeva, Inorg. Mater. (Engl. Transl.) 1990.

[28] E. Dervishi, Z. Li, F. Watanabe, A. Biswas, Y. Xu, A.R. Biris, V. Saini, A.S. Biris, Large-scale graphene

production by RF-cCVD method, Chem. Commun., DOI 10.1039/B906323D(2009) 4061-4063. 


\section{Highlights}

- Hummers synthesis consists of three stages: dissolution, intercalation and crystal.

- GO is produced early on during the synthesis and display new diffraction peaks.

- An unidentified triclinic phase is observed for the Hummers synthesis.

- Thermal reduction of GO display three stages: GO, a disordered stage and rGO.

- In situ XRD indicate reformation of rGO even for fast heated thermal reduction.

ToC

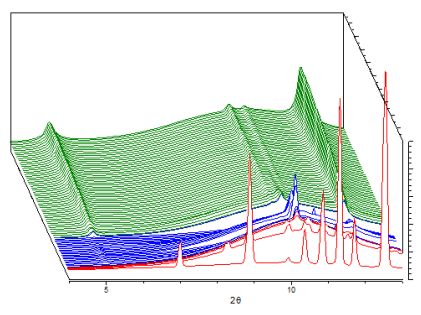

In situ X-ray diffraction results for of the modified Hummers synthesis and the thermal reduction of graphene oxide, revealing three stages for both syntheses as well as new GO diffraction peaks and unidentified crystalline material for the Hummers synthesis and a disordered stage for the thermal reduction of graphene oxide. 